\title{
Inhibition of phosphoinositide 3-kinase is associated with reduced angiogenesis and the altered expression of angiogenic markers in endothelioma cells
}

P. Mabeta

Angiogenesis Laboratory, Department of Anatomy and Physiology, University of Pretoria, Private Bag X04, Tshwane, 0110, South Africa

Corresponding Address

Dr. P. Mabeta

Angiogenesis Laboratory

P/Bag X04

Dept. of Anatomy and Physiology

University of Pretoria

Onderstepoort, Tshwane, 0110

South Africa

Tel: +27 125298354

Fax: +27 125298305

E-mail: peace.mabeta@up.ac.za 


\section{Summary}

The phosphoinositide 3-kinase (PI3k) signaling pathway is involved in the regulation of numerous cellular activities. The pathway has also been implicated in the development of various tumors. In the context of vascular tumors, the role of the PI3k signaling still needs to be established. In the present study the effects of blocking PI3k activation on endothelioma cells derived from mice with vascular tumors were investigated using the crystal violet assay, real time cell analysis, light microscopy, the aorta ring assay and antibody arrays. The suppression of PI3k led to the inhibition of cell growth, cell migration, as well as angiogenesis. The inhibition of these processes correlated with low Akt activity. Antibody array analysis revealed that there was a suppression of several proangiogenic molecules, including Eotaxin-1 and basic fibroblast growth factor (bFGF) in cultures treated with LY294,002, an inhibitor of PI3k. At the same time, LY294,002 increased the expression of platelet factor 4 (PF4), interleukin (IL)-12 p40/70 and the Fas ligand (FasL), molecules which have antiangiogenic properties. The results suggest that PI3k may play a role in the expression of some of the key regulatory molecules involved in angiogenesis, and perhaps in the growth of endotheliomas. As such, it is plausible that the PI3k/Akt pathway may be a target for therapeutic molecules designed for the treatment of endothelial tumors.

Keywords: PI3k, endothelioma, angiogenesis, Akt, LY294,002 


\section{Introduction}

Vascular tumors are endothelial cell neoplasms characterised by dysregulated angiogenesis. ${ }^{1}$ In chicks, the overexpression of $\mathrm{P} 3 \mathrm{k}$, an oncogene coding for the homologue catalytic subunit of PI3k, has been shown to lead to the development of vascular tumours known as hemangiosarcomas. ${ }^{2}$ In a recent study, we showed that the growth of vascular tumors in mice correlated with increased Akt activation. ${ }^{3}$ The significance of the PI3k-Akt pathway in the pathogenesis of vascular tumors requires elucidation, and the key role players in PI3k signaling need to be established.

The phosphoinositide 3-kinases, also known as phosphatidylinositol-3-kinases (PI3ks) are a family of enzymes that regulate a network of cellular processes. ${ }^{4-6}$ In mammalian cells, PI3ks exist in three classes, I, II, and III, with class I being the most studied in the context of the regulation of cell growth, survival and proliferation. ${ }^{5-7}$ Class I PI3ks are further divided into the IA and IB subgroups based on the receptors that activate them. ${ }^{5,7}$ The former, which transmits signals from receptor tyrosine kinases (RTKs), has been implicated in tumorigenesis and is thus the focus of attention in this study.

Upon binding of a growth factor or signaling compound to a receptor tyrosine kinase (RTK), PI3k is recruited to the internal site of the cell membrane and activated. ${ }^{5}$ Such activation produces 3 ' phosphoinositide lipids which act as second messengers by activating diverse cellular target molecules. ${ }^{5,7}$ One such example is the phosphorylation of phosphatidylinositol 4,5-bisphosphate (PIP2) by PI3ks to form phosphatidylinositol 3,4,5trisphosphate (PIP3). ${ }^{5}$

Phosphatidylinositol 3,4,5-trisphosphate initiates a cascade of events that lead to the recruitment and activation of protein kinase $\mathrm{B}$ (PKB), also known as Akt. The protein kinase is one of the key downstream effectors of PI3k. When activated, Akt regulates a broad array of cellular functions, including cell growth, attachment, proliferation, migration, and apoptosis. On the other hand, the subversion of the functions of PI3k is observed in a myriad of disorders that include cancer, diabetes mellitus and inflammation. ${ }^{6}$ Following the establishment of the importance of PI3k in physiological processes and in disease, a number of inhibitors, most notably Wortmannin and LY294,002, were developed to aid in studying the role of PI3k in the different settings. LY294,002 is a derivative of the broad spectrum kinase inhibitor quercetin and unlike Wortmannin, it's effects are reversible. ${ }^{6}$ 
In this study, we investigated the effects of LY294002 on endothelioma (sEnd.2) cells, which are endothelial cells derived from the subcutaneous tissue of mice with Pym Tinduced vascular tumors. It is envisaged that better understanding of the involvement of the PI3k signaling pathway in vascular tumor growth may provide useful targets for therapeutic intervention. In the present study, the effects of the drug on endothelioma cell growth and migration, and on angiogenesis and the expression of angiogenic markers were investigated.

\section{Materials and Methods}

2.1 Cell culture maintenance procedure

Endothelioma cells (obtained from Prof M.S. Pepper, University of Pretoria) were maintained at a temperature of $37^{\circ} \mathrm{C}$ in a humidified atmosphere containing $5 \% \mathrm{CO}_{2}$. The cells were cultured in Dulbecco's modified eagle's medium (Sigma-Aldrich, St. Louis, MO, USA), supplemented with $10 \%$ heat inactivated fetal calf serum (Invitrogen, USA), $2 \mathrm{mM} \mathrm{L-}$ glutamine (Invitrogen, CA, USA), and 1\% Penicillin-streptomycin (Whitehead Scientific, Johannesburg, South Africa).

\subsection{Cell growth assay}

Cell viability was assessed using the crystal violet nuclear staining assay. Endothelioma cells were seeded in 24-well culture plates at a density of 10000 cells per well for 24 hours, and then treated with LY294,002 (0-10 $\mu \mathrm{M})$ or dimethylsulfoxide (DMSO) for 24-72 hours. At termination cells were fixed with $1 \%$ glutaraldehyde in PBS for 15 minutes, followed by staining with a $0.1 \%$ crystal violet solution (Sigma-Aldrich, St Louis, MO, USA) for 30 minutes. The chromophore was extracted with 0.1\% Triton X-100 (Sigma-Aldrich, St Louis, MO, USA) per well. The absorbance was read at $570 \mathrm{~nm}$ on an ELx 800 Universal Microplate Reader (Bio-Tek instruments Inc, Weltevreden, SA). Three wells were analysed for each concentration. The percentage of viable cells was calculated as follows: viability (\%) $=[$ A570(treated $)-$ A570(blank) $] /[$ A570(control) - A570(blank) $]$ x 100.

\subsection{Cell Morphology}

Endothelioma cells were seeded on cover slips in 6-well plates at a density of $3 \times 10^{5}$. After 24 hours, the cells were treated with LY294,002 $(0-10 \mu \mathrm{M})$ over a period of 48 hours. The cells were fixed in Bouin's fluid. After 30 minutes of fixing they were rinsed in $70 \%$ ethanol for a further thirty minutes, and then rinsed with water. 
The cells were immersed in Meyer's haemalum for twenty minutes, rinsed with water, followed by $70 \%$ ethanol. The cells were incubated in $1 \%$ eosin for two minutes and then rinsed twice with increasing concentrations of ethanol (70\%, 96\%, and 100\%). Xylol was used to rinse the cells twice before mounting them onto microscopic glass slides using resin. The slides were viewed with an Olympus BX63 light microscope and images were captured using the Olympus DP72 digital camera (Wirsam Scientific, Johannesburg South Africa).

\subsection{Caspase activity}

Endothelioma cells were seeded at a density of $3 \times 10^{5}$ cells/well and allowed to attach overnight. The cells were treated with LY294,002 $(10 \mu \mathrm{M})$ with or without the caspase inhibitor zVADfmk $(50 \mu \mathrm{M})$ for 48 hours. Control cells were exposed to DMSO. Caspase-3 activity was measured using commercially available kits according to the manufacturer's instructions (BioVision, Mountain View, USA). In brief, control and drug-treated sEnd.2 cells were lysed in caspase lysis buffer, followed by centrifugation at 16,0009g for $15 \mathrm{~min}$. The supernatant was mixed with assay buffer in the presence of $200 \mathrm{mM}$ substrate. Samples were incubated at $37 \mathrm{oC}$ for $4 \mathrm{~h}$ and absorbance was read at $405 \mathrm{~nm}$ using an ELx 800 Microplate Reader (Bio-Tek instruments Inc, Weltevreden, SA).

\subsection{Akt kinase activity}

The activity of Akt kinase was measured in control and LY294,002-treated cells using an enzyme-linked immunosorbent assay (ELISA) kit according to the instructions of the manufacturer (Stressgen, MI, USA). Cells were seeded at a density of $3 \times 10^{5}$ cells/well. After 24 hours the cells were treated with LY294,002 at the indicated doses or with DMSO (control). After 48 hours, $50 \mu \mathrm{L}$ of assay buffer was added to each well. Thirty $\mu$ l of sample, purified active Akt, inhibitor diluent or dilution buffer were added to triplicate wells. Ten $\mu 1$ of diluted ATP were then added and the plate incubated for 90 min. Anti-phosphate specific substrate was added to each well and the plate was incubated at room temperature. This was followed by the addition of $40 \mu \mathrm{L} /$ well of secondary antibody solution. The plate was incubated at room temperature for $30 \mathrm{~min}$. After washing, tetramethylbenzidine was added to each well and the plate was incubated at room temperature for $45 \mathrm{~min}$. The reaction was stopped by adding $20 \mu \mathrm{L} /$ well of acid stop solution. The absorbance was read at $450 \mathrm{~nm}$ using the ELx 800 Universal Microplate Reader (Bio-Tek instruments Inc, Weltevreden, SA). 


\subsection{Cell Migration Studies}

Cell migration experiments were performed using 16-well plates (CIM-16, Roche Applied Science, Johannesburg, South Africa). Prior to each experiment, cells were deprived of FBS for 24 hours. A volume of $160 \mu \mathrm{L}$ serum free medium with or without $10 \mathrm{ng} / \mathrm{ml}$ bFGF as a chemoattractant was added to each well of the lower chamber of a CIM plate. Serum free medium $(20 \mu \mathrm{L})$ was also added to the upper chamber and the plate was allowed to equilibrate for 1 hour. Endothelioma cells $\left(6 \times 10^{3}\right.$ cells $)$ were seeded into the upper chamber of the plates and treated with LY294,002 at the indicated concentrations. Identical amounts of dimethyl sulfoxide were used as control. Each condition was performed in quadruplicate. The cells that migrated through the filter into the bottom chamber were counted with an xCELLigence RTCA DP instrument. The cell index (CI) was recorded by the instrument analyzer and analysis was performed with the supplied RTCA software (vs. 1.2.1). Results are based on raw data without CI-normalization.

\subsection{Aorta ring assay}

Aortas were harvested from 6-8 weeks old male Sprague-Dawley rats, cleaned, and cut into $1 \mathrm{~mm}$ wide rings. The rings were then embedded in fibrin gel in 24 -well culture plates and maintained in MCDB-131 medium supplemented with $0.3 \mathrm{mg} / \mathrm{ml}$ amino caproic acid and 1\% penicillin-streptomycin. The cultures were treated with either LY294,002 at the indicated doses or DMSO for 10 days. Medium and drug were renewed every 2 days. Cultures were monitored for growth every second day with a Zeiss Axiovert microscope (Carl Zeiss, Jena, Germany) attached to a digital camera. The area of neovessel growth was determined using Ethical approval was obtained from the University of Pretoria's Animal Use and Care Committee (project V025-08).

\subsection{Antibody Arrays}

Endothelioma cells were seeded at a density of $2.5 \times 10^{5}$ cells. After 24 hours, the cells were treated with LY-294,002 or DMSO over a period of 48 hours. The mouse angiogenesis array kit which employs a membrane spotted with 24 proteins in duplicate (RayBiotech Inc., Norcross, USA) was used to study the expression profile of angiogenic proteins in the conditioned medium of control and LY-294,002-treated cells according to the protocol of the manufacturer. Protein expression was visualized with a FluorChem Imaging System (Proteinsimple, CA, USA). 


\subsection{Data Analysis}

Statistics were performed using one-way ANOVA, and where statistical significance was reached, pair-wise multiple comparisons were made using an ad hoc test (Bonferroni's method). Significance was designated as $\mathrm{P}<0.05$.

\section{Results}

\subsection{Cell growth}

The effects of LY294,002 on endothelioma (sEnd.2) cell growth were evaluated over 72 hours. LY294,002 induced a decrease in sEnd.2 cell numbers in a dose-dependent and time-dependent manner (Fig 1A). The drug was less effective over 24 hours of treatment, while about $80 \%$ of the cells died after 72 hours of treatment (Fig 1A). The $\mathrm{IC}_{50}$ values were approximately $9.80,2.40$ and $0.12 \mu \mathrm{M}$ over 24,48 and 72 hours respectively.

\subsection{Cell Morphology}

Following the observation that LY294,002 inhibited the growth of endothelioma cells, the effect of the drug on cell morphology was studied using light microscopy. Control cultures stained with $\mathrm{H} \& \mathrm{E}$ showed cells in different stages of mitosis (Fig 1B). In LY294,002-treated cultures, cells appeared to have lost cell-cell contacts, and some of the cells had condensed chromatin, indicating possible cell death (Fig 1C).

\subsection{Caspase activity}

To determine possible apoptosis induction, Caspase 3 activity was measured using ELISA. As a control measure, the caspase inhibitor zVAD fmk was included. Caspases are a family of death cysteine proteases; in particular, caspase 3 is an executioner caspase which can be activated by either the extrinsic or the intrinsic apoptotic pathways. ${ }^{8}$ In most cell types it is required for the typical morphological and biochemical changes associated with apoptosis, such as membrane blebbing, chromatin condensation and DNA fragmentation. ${ }^{9}$ The results in this study showed that there was an increase in the activation of Caspase- 3 in endothelial cell cultures following LY294,002 treatment compared to the DMSO control $(\mathrm{P}<0.001)$. Such an increase in caspase-3 activation was not observed in cells treated with a combination of LY294,002 and the broad spectrum caspase inhibitor zVAD fmk (Fig. 2). Therefore these observations indicate that LY294,002 induced an increase in caspase 3 activity in these endothelial cells. 


\subsection{Akt kinase activity}

Activated Akt has been shown to play a role in the regulation of apoptotic factors such as caspases. ${ }^{8}$ The activity of Akt was measured to determine if it was affected in LY294,002-treated cells given the relationship between caspase activation and Akt activity. The results showed that the levels of Akt kinase activity were lower in cells treated with the PI3K inhibitor LY294,002 than in control cells (Fig. 3). In addition to its role in the inhibition of apoptosis, increased activity of Akt is associated with increased cell survival, and with the promotion of cell migration and angiogenesis. ${ }^{10}$

\subsection{Cell Migration Studies}

To investigate the effects of PI3k inhibition on endothelioma cell migration, cells were seeded in serum free medium in the upper chambers of CIM-plates, while bFGF was used as a chemoattractant in the lower chambers of the plates. The real-time migration of endothelioma cells was monitored over 21 hours by measuring electrical impedance using the xCELLIgence system. The drug LY294,002 inhibited cell migration in a dose dependent manner (Fig. 4).

\subsection{Aorta ring assay}

Previously, studies revealed that these endothelioma cells form cysts when cultured in a gel matrix, and not tubular structures as seen with most endothelial cells. ${ }^{11}$ This characteristic was attributed to the increased proteolytic activity observed with these cells. ${ }^{11}$ Given that endothelioma cells do not form capillary-like structures in vitro, angiogenesis was evaluated using the aorta ring assay. In control cultures, aorta rings gave rise to an array of neovessels (Fig.5A). However, the area of neovessel growth was reduced in cultures treated with LY294,002 (Fig. 5B).

\subsection{Antibody Array Studies}

Angiogenic protein expression was determined in conditioned medium using an angiogenesis antibody array kit (RayBiotech Inc. GA, USA). Reduced expression of bFGF and Eotaxin were observed in conditioned medium from LY294,002-treated cells (Fig. 6). Both leptin and VEGF were not detected in drug treated cultures, whereas faint spots representing these proteins were observed in the control (Fig. 6). It appears that LY294,002 inhibited VEGF and leptin expression. An increase in the expression of IL-12p40/70, PF4 and the FasL were observed following the treatment of cells with the drug. 


\section{Discussion}

The PI3k/Akt pathway has been shown to contribute to tumor growth, and to be activated in several cancers. ${ }^{12}$ In a recent study, Rapamycin, which regulates Akt phosphorylation, induced a decrease in vascular tumor growth and inhibited the phosphorylation of Akt in the same neoplastic cells. ${ }^{1}$ In another study the inhibition of vascular tumor growth was associated with a decrease in Akt activity. ${ }^{5}$ In the current study the suppression of PI3k, and ultimately Akt, lead to the inhibition of endothelioma cell growth and migration. As with previous studies on other angiogenesis models, ${ }^{13,14}$ our data revealed that PI3k inhibition led to a decrease in neovessel formation in the aorta ring assay. Indeed past studies have shown that constitutively active PI3k induced angiogenesis, and that when activated, PI3k played a role in the regulation of downstream molecules that are involved in angiogenesis. ${ }^{4,15}$

Other studies have indicated that the inhibition of PI3k leads to a decrease in both tumor growth and angiogenesis. ${ }^{4,13}$ In the current study, morphological analysis of endothelial cells treated with the drug revealed features of apoptosis. This is consistent with our observation of increased caspase activity. Given that PI3k-Akt promotes cell survival, it is not surprising that its inhibition is linked with the induction of apoptosis. The inhibition of apoptosis is also essential during angiogenesis, while the induction of endothelial cell apoptosis disrupts angiogenesis. ${ }^{16}$ A key finding of this study was that the suppression of PI3k altered the expression of several angiogenic markers in endothelioma cultures. There was an increase in the expression of Fas ligand (FasL) in treated cultures. The Fas ligand is a type-II transmembrane protein that belongs to the tumor necrosis factor (TNF) family. ${ }^{17}$ It's binding to the cell-surface Fas receptor (Fas), induces a pro-apoptotic signaling cascade which leads to caspase activation and ultimately cell death. ${ }^{18}$ Earlier studies have revealed that anti-FasL inhibited endothelial cell apoptosis and promoted angiogenesis in vitro, while in vivo studies have shown that FasL deficient mice have an increased incidence of angiogenesis. ${ }^{17}$ Another factor that exhibited increased expression following LY294,002treatment is Platelet factor 4 (PF4), also known as CXCL4. It is a 70-amino acid molecule which belongs to the CXC chemokine family. ${ }^{19,20}$

It is released by alpha-granules of activated platelets during platelet aggregation and it promotes coagulation. It is also a chemoattractant for neutrophils and fibroblasts. ${ }^{21}$ In addition to its role in coagulation, PF4 appears to have antiangiogenic effects. ${ }^{20,21}$

In vitro, it inhibits endothelial cell proliferation and migration, and in vivo it suppresses 
angiogenesis. $^{21}$

One of the mechanisms that have been proposed to explain PF4's antiangiogenic effects is possible binding to heparin proteoglycans, which in turn interferes with angiogenic growth factor activity. Another proposed mechanism is through direct interaction with angiogenic growth factors such as VEGF. ${ }^{20}$

In comparison with LY294,002-treated cultures, there was increased bFGF and eotaxin-1 expression in control cultures. Also, faint spots representing VEGF and leptin were observed in control cultures, while these factors were not expressed in LY294,002-treated cultures. Several lines of research have established that VEGF and bFGF stimulate angiogenesis. These growth factors have also been shown to stimulate tumor angiogenesis. ${ }^{22}$ Eotaxin-1, also known as CCL-11 was reported to promote angiogenesis and cancer metastasis. ${ }^{23,24}$ Another factor that appeared to be inhibited by LY294,002 is leptin, a 16$\mathrm{kDa}$ adipokine that plays a key role in regulating energy intake and expenditure. ${ }^{25}$ It is secreted mainly by white adipose tissue. ${ }^{26}$ Leptin has also been reported to promote endothelial cell proliferation and to upregulate both VEGF and its receptor, vascular endothelial receptor 2 (VEGFR2). ${ }^{27-29}$ Thus LY294,002 appears to suppress proangiogenic factors and chemokines while stimulating the expression of antiangiogenic molecules.

In conclusion, these findings suggest that the PI3k-Akt pathway might play an important role in angiogenesis and in endothelioma growth. Further studies are warranted to establish the role of the components of the pathway as potentially useful therapeutic targets in vascular tumors, particularly endotheliomas.

\section{Disclosure of interest}

The author declares that there is no conflict of interest to disclose with regard to this article.

\section{Acknowledgements}

This study was supported by the University of Pretoria and the National Research Foundation (NRF). 


\section{References}

[1] Du W, Gerald D, Carole A Perruzzi CA, Rodriguez-Waitkus P, Enayati L, Krishnan B, Edmonds J, Hochman LM, Lev DC, Phung TL. Vascular tumors have increased p70 S6Kinase activation and are inhibited by topical Rapamycin. Lab Investigation 2013;93:11151127.

[2] Jiang BH, Zheng JZ, Aoki M, Vogt PK. Phosphotidylinositol 3-kinase signaling mediates angiogenesis and expression of vascular endothelial growth factor in endothelial cells. PNAS 2000;97:1749-1753.

[3] Mabeta P, Pepper MS. Inhibition of hemangioma development in a syngeneic mouse model correlates with Bcl-2 suppression and the inhibition of Akt kinase activity.

Angiogenesis 2012;15:131-139.

[4] Vivanco I, Sawyers CS. The Phosphotidylinositol 3-Kinase-Akt Pathway in human cancer. Nature Reviews Cancer 2002;2:489-501.

[5] Gharbi SI, Zvelebil MJ, Shuttleworth SJ, Hancox T, Saghir N. Exploring the specificity of the PI3K family inhibitor LY294002. Biochem J 2007;404:15-21.

[6] Dituri F, Mazzocca A, Giannelli G, Antonaci S. PI3K functions in cancer progression, anticancer immunity and immune evasion by tumors. Clin Dev Immunol 2011;2011:947858947958.

[7] Gerard N, Caillaud M, Martoriati A, Goudet G, Lalmanach A. The Interleukin-I system and female reproduction. J Endocrinol 2004;180:203-212.

[8] Moon D-O, Park S-Y, Choi YH, Kim ND, Lee C, Kim G-Y. Melittin induces Bcl-2 and Caspase-3-dependent apoptosis through downregulation of Akt phosphorylation in human leukemic U937 cells. Toxicon 2008;51:112-120.

[9] Porter AG, Janicke RU. Emerging roles of Caspase-3 in apoptosis. Cell Death Differ 1999;6:99-104.

[10] Okumura N, Yoshida H, Kitagishi Y, Murakami M, Nishimura Y, Matsuda S. $\mathrm{PI} 3 \mathrm{~K} / \mathrm{AKT} / \mathrm{PTEN}$ signaling as a molecular target in leukemia angiogenesis. Adv Hematol 2012;1:1-6.

[11] Montesano R, Pepper MS, Möhle-Steinlein U, Risau W, Wagner EF, Orci L. Increased Proteolytic Activity is responsible for the aberrant morphogenetic behavior of endothelial cells expressing the Middle T Oncogene. Cell 1990;62:435-445.

[12] Katso R, Okkenhaug K, Ahmadi K, White S, Timms J, Waterfield MD. Cellular function of Phosphoinositide 3-kinases: Implications for development, homeostasis, and cancer. Annu Rev Cell Dev Biol. 2001;17:615-675.

[13] Graupera M, Potente M. Regulation of angiogenesis by PI3k signaling networks. Exp Cell Res 2013;319:1348-1355. 
[14] Jiang BH, Liu LZ. PI3K/PTEN signaling in angiogenesis and tumorigenesis . Adv Cancer Res 2008;102:150-158.

[15] Shiojima I, Walsh K. Role of Akt signaling in vascular homeostasis and angiogenesis. Circ Res 2002;90:1243-1250.

[16] Mabeta P, Pepper MS. A comparative study on the anti-angiogenic effects of DNAdamaging and cytoskeletal-disrupting agents. Angiogenesis 2009;12:18-90.

[17] Kaplan HJ, Leibole MA, Tezel T, Ferguson TA. Fas Ligand (CD95 Ligand) controls angiogenesis beneath the retina. Nature Medicine 1999;5:292-297.

[18] Voss M, Lettau M, Paulsen M, Janssen O. Posttranslational regulation of Fas ligand function. Cell Communication and Signaling 2008;6:11-23.

[19] Bikfalvi A. Platelet Factor 4: An inhibitor of angiogenesis. 2004 30:379-85 Semin Thromb Hemost. 2004;30:379-385.

[20] Bikfalvi A. Recent developments in the inhibition of angiogenesis: Examples from studies on Platelet Factor-4 and the VEGF/VEGFR system. Biochem Pharmacol 2004;68:1017-1021.

[21] Aidoudi S BA. Interaction of PF4 (CXCL4) with the vasculature: A role in atherosclerosis and angiogenesis. Thromb Haemost 2010;104:941-948.

[22] Cross MJ CL. FGF and VEGF function in angiogenesis: Signalling pathways, biological responses and therapeutic inhibition. Trends Pharmacol Sci 2001;22:201-207.

[23] Levina V, Nolen BM, Marrangoni AM, et al. Role of Eotaxin-1 signaling in ovarian cancer. Clinical Cancer Research 2009; 15:2647-2656.

[24] Salcedo R, Young HA, Ponce ML, Ward JM, Kleinman HK, Murphy WJ, Oppenheim JJ. Eotaxin (CCL11) induces in vivo angiogenic responses by human CCR3+ endothelial cells . J Immunol 2001;166:7571-7578.

[25] Houseknecht KL, Baile CA, Matteri RL, Spurlock ME. The biology of Leptin: A Review. J Animal Sci1998;76:1405-1420.

[26] Margetic S, Gazzola C, Pegg GG, Hill RA. Leptin: A Review of its peripheral actions and interactions. Int J Obesity 2002;26:1407-1433.

[27] Gonzalez-Perez RR, Lanier V, Newman G. Leptin's pro-angiogenic signature in breast cancer. Cancers 2013;5:1140-1162.

[28] Vona-Davis L, Rose DP. Angiogenesis, adipokines and breast cancer. Cytokine Growth Factor Rev 2009;20:193-201.

[29] Kelesidis T, Kelesidis I, Chou S, Mantzoros CS. Narrative Review: The role of leptin in human Physiology: Emerging clinical applications. Annals of Int Med 2010;152:93-100. 


\section{Figure Legends}

Figure 1. (A) Cell viability was determined in control and LY294,002-treated sEnd.2 cells using crystal violet staining. Three wells were analysed per drug concentration. Results are expressed as mean percentage of cells relative to control $\pm \mathrm{SD}$ of three separate experiments. $* \mathrm{P}<0.05$ (B-C) Representative micrographs showing the effects of LY294,002 on endothelioma cell morphology. Light microscopy was used to analyse the H \& E stained cells. (B) In control cultures endothelioma cells formed a monolayer and cells in different stages of mitosis, including metaphase and telophase were observed. (C) In drug-treated cultures, and the cells were round, appeared to have lost cell-cell contacts, and had condensed chromatin. Bar: $20 \mu \mathrm{m}$

Figure 2. LY294,002 increases the activity of caspases-3 in endothelioma cells. Cytosolic lysates were prepared from DMSO- or LY294,002-treated sEnd.2 cells. Caspase-3 activation was determined using a commercially available ELISA kit according to the manufacturer's instructions. Three wells were analyzed per treatment dose. Values (in arbitrary units) are mean \pm SD from three experiments. $\S \mathrm{P}<0.05 ; * * \mathrm{P}<0.001$

Figure 5. Inhibition of angiogenesis by LY294,002 in a rat aorta ring assay. Cultures were monitored every second day and representative images were photographed (A) Control culture showing an aorta ring with microvessels growing from its surface; (B) An aorta ring treated with $10 \mu \mathrm{M}$ LY294,002. Bar $250 \mu \mathrm{m}$. (C) Quantitative analysis of in vitro angiogenesis was undertaken by calculating the means of the vascularised areas. Each data point represents the mean \pm SD of three wells from two separate experiments.

Figure 3. The effects of LY294,002 on Akt activity in sEnd.2 cells. Akt activity was measured using ELISA. Data are expressed as the mean \pm SD of triplicate wells. The experiment was repeated. $* * \mathrm{P}<0.001$

Figure 4. Migration assay of endothelioma cells treated with LY294,002. Cells were seeded in RTCA CIM-16 plates in serum free medium, and bFGF was used as a chemoattractant. Impedance measurements were performed over 21 hours using the xCELLIgence RTCA instrument. Four wells were analysed per treatment condition. Data (in mean CI \pm SD) are from two separate experiments. 
Figure 6. Chemiluminiscent detection of angiogenic proteins using antibody arrays. The expression of proteins on membranes was investigated in the conditioned medium of control and LY294,002-treated endothelioma cells using the mouse angiogenesis antibody array kit (RayBiotech, Inc. Norcross, USA). The study was repeated. The angiogenic factors and cytokines are listed in the table. 
Figures

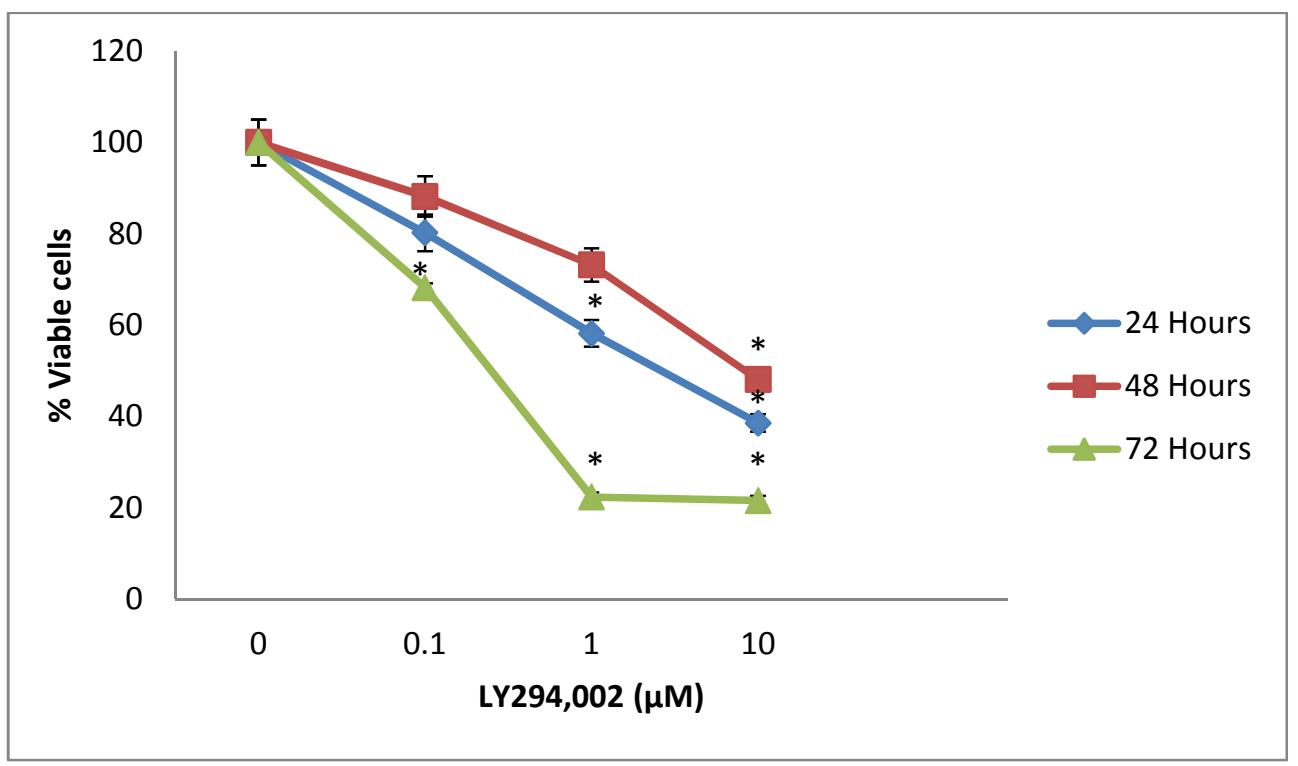



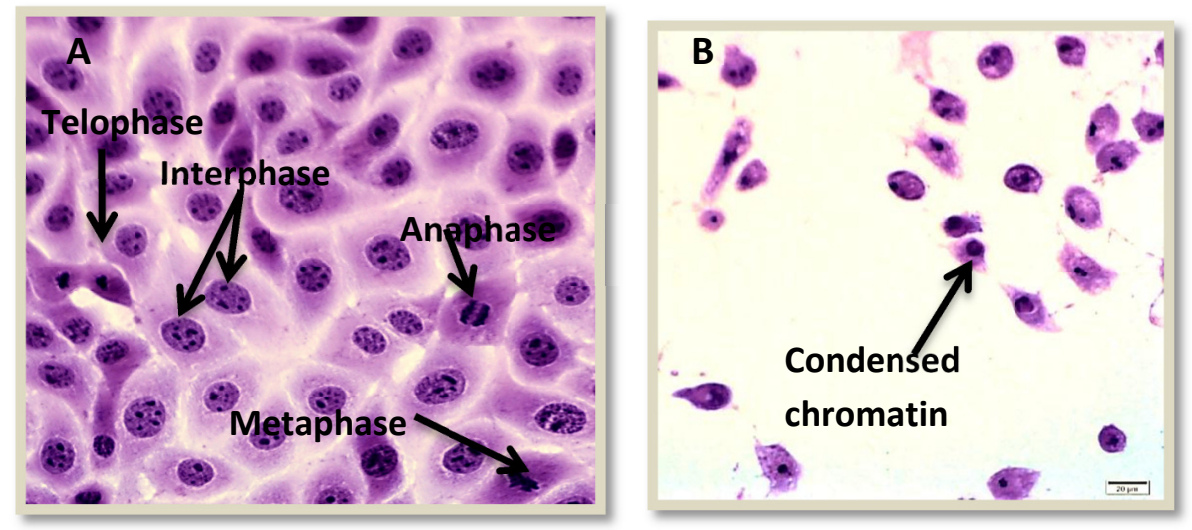


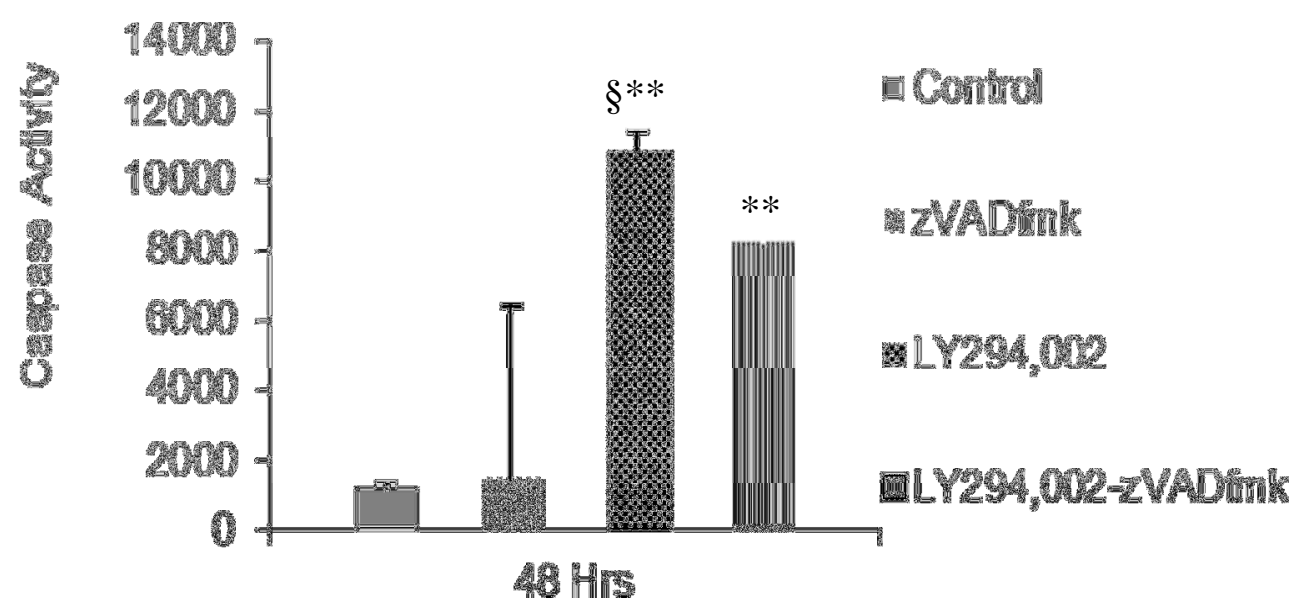




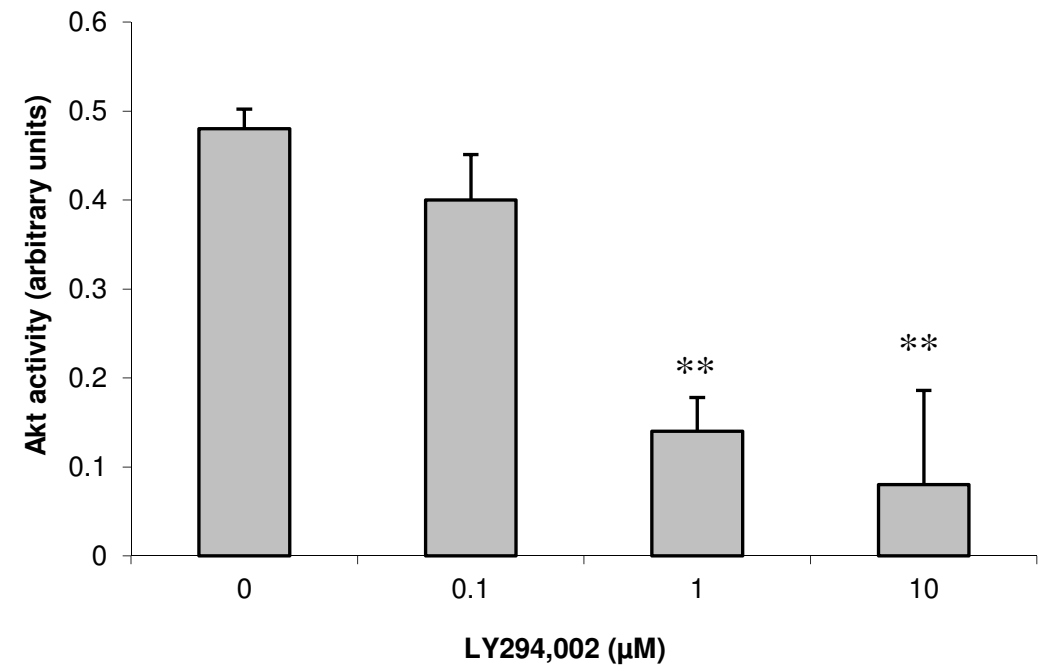




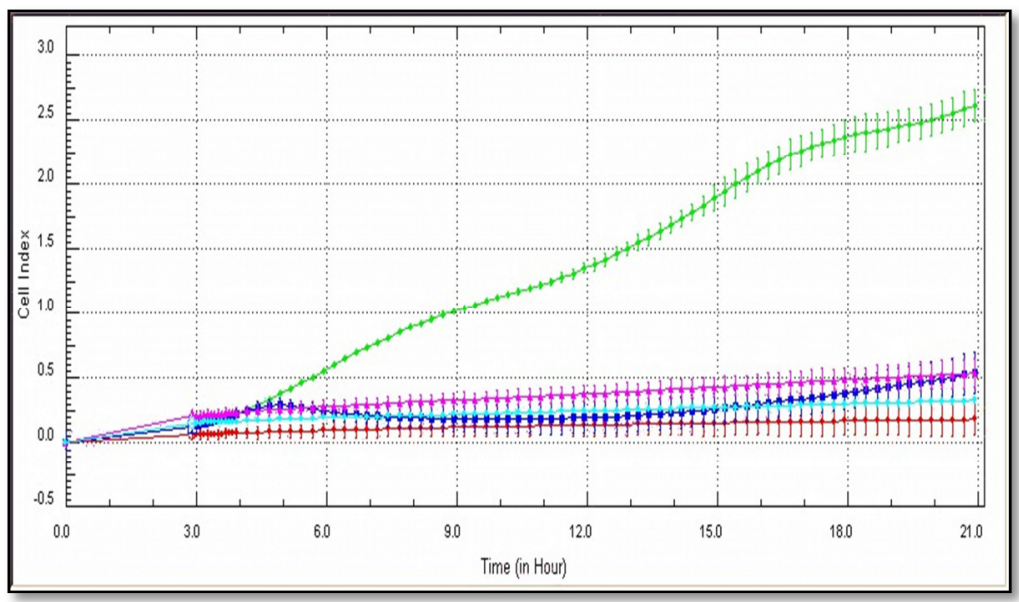

Contro

$-0.01 \mu \mathrm{M}$ LY294,002

$-0.1 \mu \mathrm{M}$ LY294,002

1 MM LY294,002

$-10 \mu \mathrm{M}$ LY294,002 

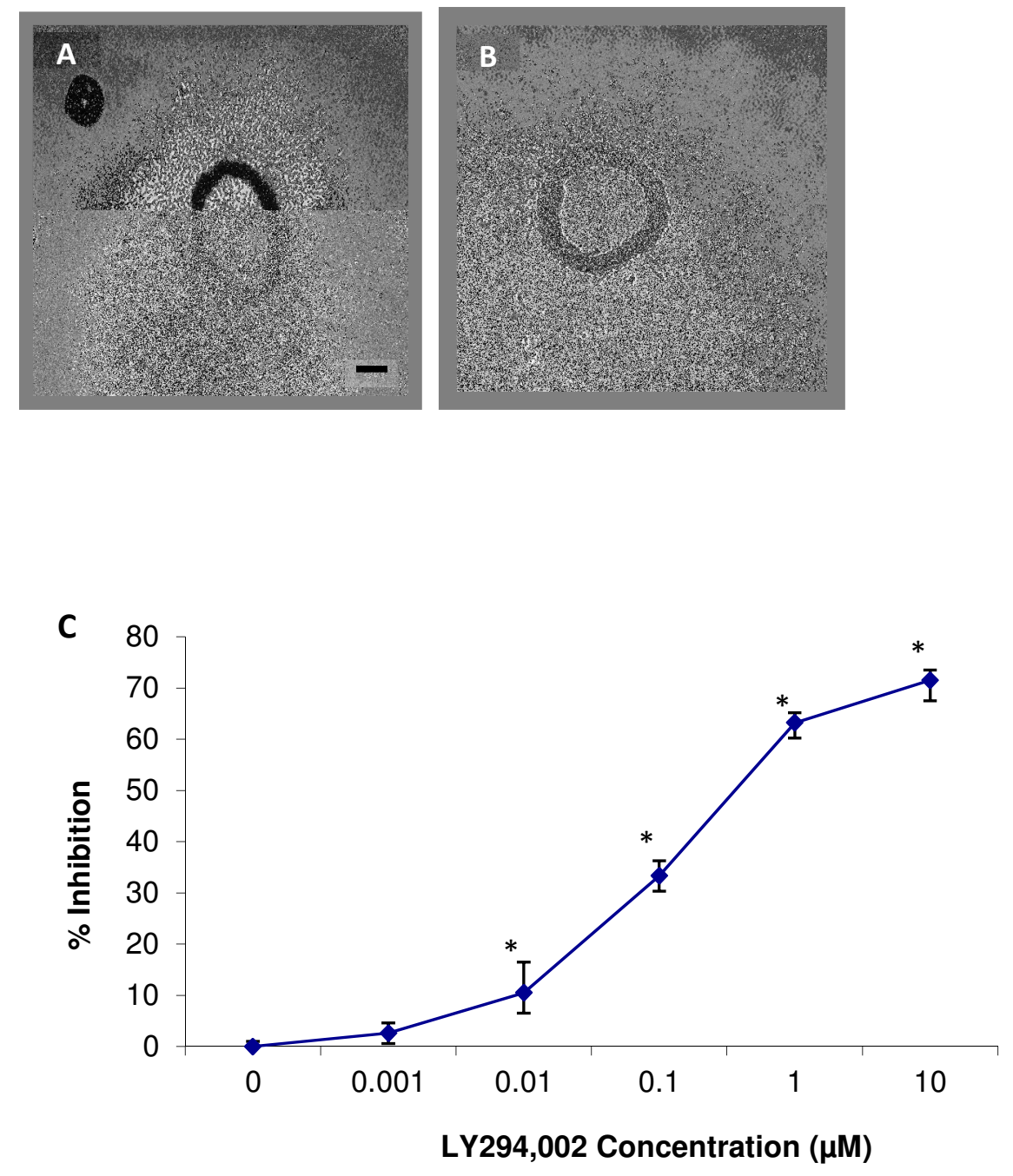


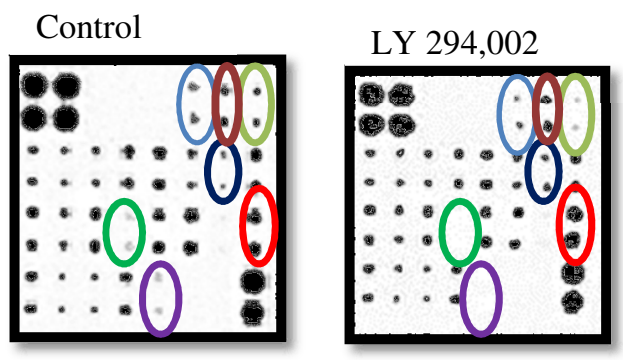

\begin{tabular}{|c|c|c|c|c|c|c|c|c|}
\hline & A & B & C & D & E & F & G & H \\
\cline { 2 - 9 } 1 & POS & POS & NEG & NEG & BLANK & Eotaxin-1 & Fas Ligand & bFGF \\
\hline 2 & POS & POS & NEG & NEG & BLANK & Eotaxin-1 & Fas Ligand & bFGF \\
\hline 3 & G-CSF & GM-CSF & $\begin{array}{c}\text { IFN } \\
\text { gamma }\end{array}$ & IGF-2 & IL-1 alpha & IL-1 beta & IL-12p40/70 & IL-12p70 \\
\hline 4 & G-CSF & GM-CSF & $\begin{array}{c}\text { IFN } \\
\text { gamma }\end{array}$ & IGF-2 & IL-1 alpha & IL-1 beta & IL-12p40/70 & IL-12p70 \\
\hline 5 & IL-13 & IL-6 & IL-9 & Leptin & MCP-1 & M-CSF & MIG & PF-4 \\
\hline 6 & IL-13 & IL-6 & IL-9 & Leptin & MCP-1 & M-CSF & MIG & PF-4 \\
\hline 7 & TIMP-1 & TIMP-2 & TNF-alpha & THPO & VEGF & BLANK & BLANK & POS \\
\hline 8 & TIMP-1 & TIMP-2 & TNF-alpha & THPO & VEGF & BLANK & BLANK & POS \\
\hline
\end{tabular}

POS $=$ Positive control spot NEG = Negative control spot; BLANK = Blank spot 